\title{
PET/MRI and PET/CT in follow-up of head and neck cancer patients
}

\author{
Marcelo A. Queiroz • Martin Hüllner • Felix Kuhn • \\ Gerhardt Huber • Christian Meerwein • Spyros Kollias • \\ Gustav von Schulthess $\cdot$ Patrick Veit-Haibach
}

Received: 20 September 2013 / Accepted: 16 January 2014 / Published online: 28 February 2014

(C) Springer-Verlag Berlin Heidelberg 2014

\begin{abstract}
Purpose Positron emission tomography (PET)/MRI combines the functional ability of PET and the high soft tissue contrast of MRI. The aim of this study was to assess contrastenhanced (ce)PET/MRI compared to cePET/CT in patients with suspected recurrence of head and neck cancer (HNC). Methods Eighty-seven patients underwent sequential cePET/ CT and cePET/MRI using a trimodality PET/CT-MRI set-up. Diagnostic accuracy for the detection of recurrent HNC was evaluated using cePET/CT and cePET/MRI. Furthermore, image quality, presence of unclear ${ }^{18} \mathrm{~F}$-fluorodeoxy-D-glucose (FDG) findings of uncertain significance and the diagnostic advantages of use of gadolinium contrast enhancement were analysed.
\end{abstract}

M. A. Queiroz $\cdot$ M. Hüllner · F. Kuhn • G. von Schulthess ·

P. Veit-Haibach

Department of Medical Radiology, Nuclear Medicine, University

Hospital Zurich, Zurich, Switzerland

F. Kuhn $\cdot$ P. Veit-Haibach

Department of Medical Radiology, Diagnostic and Interventional

Radiology, University Hospital Zurich, Zurich, Switzerland

M. Hüllner · S. Kollias

Department of Medical Radiology, Neuroradiology, University

Hospital Zurich, Zurich, Switzerland

G. Huber $\cdot$ C. Meerwein

Department of Otorhinolaryngology, University Hospital Zurich, Zurich, Switzerland

M. A. Queiroz $(\square)$

Department of Nuclear Medicine, University Hospital Zurich, 8091 Zurich, Switzerland

e-mail: marcelo.araujoqueiroz@usz.ch

M. A. Queiroz

e-mail: marceloqueiroz10@yahoo.com.br
Results cePET/MRI showed no statistically significant difference in diagnostic accuracy compared to cePET/CT (91.5 vs $90.6 \%)$. Artefacts' grade was similar in both methods, but their location was different. cePET/CT artefacts were primarily located in the suprahyoid area, while on cePET/MRI, artefacts were more equally distributed among the supra and infrahyoid neck regions. cePET/MRI and cePET/CT showed 34 unclear FDG findings; of those 11 could be solved by cePET/MRI and 5 by cePET/CT. The use of gadolinium in PET/MRI did not yield higher diagnostic accuracy, but helped to better define tumour margins in $6.9 \%$ of patients.

Conclusion Our data suggest that cePET/MRI may be superior compared to cePET/CT to specify unclear FDG uptake related to possible tumour recurrence in follow-up of patients after HNC. It seems to be the modality of choice for the evaluation of the oropharynx and the oral cavity because of a higher incidence of artefacts in cePET/CT in this area mainly due to dental implants. However, overall there is no statistically significant difference.

Keywords PET/MRI - PET/CT · Head and neck cancer · Follow-up $\cdot$ Contrast media

\section{Introduction}

A concise follow-up of head and neck cancer (HNC) plays a key role in patient's survival and requires a multimodality approach, including evaluation by clinical examination, histopathology and imaging evaluation. Local recurrence is the major cause of treatment failure in patients with $\mathrm{HNC}$ after definitive therapy [1]. Conventional imaging modalities, such as contrast-enhanced computed tomography (ceCT) and contrast-enhanced magnetic resonance imaging (ceMRI), play a major role in the evaluation of locoregional 
recurrence. Both methods use mainly morphological criteria for malignancy. Treatment options (mainly radiotherapy and surgery), however, may result in anatomical distortions, scarring and post-radiation-induced inflammation, rendering distinction of active tumour tissue difficult, even if morphological assessment demonstrates a reduction in tumour or lymph node size [2]. It is less the tumour than the estimation of residual active tumour tissue that impacts on further therapeutic decisions [3].

About a decade ago, ${ }^{18}$ F-fluorodeoxy-D-glucose (FDG) positron emission tomography (PET)/CT was introduced into routine clinical practice and currently is considered a main diagnostic imaging tool for follow-up of HNC as well. It combines the functional information of PET with the morphological features of ceCT for the imaging of tumours with increased glucose metabolism [4-6]. However, PET/CT has some disadvantages, such as the use of ionizing radiation, partly reduced image quality from metal dental artefacts and it partly needs iodinated contrast for higher diagnostic overall specificity. Moreover, it has limited ability to identify cystic/necrotic lymph nodes and to thoroughly assess infiltration of neighbouring structures, mainly perineural spread [7]. Furthermore, PET/CT has a limited role in the evaluation of locoregional recurrence in the first weeks after radiation therapy. There is general consensus to set the optimum time for PET/CT to 8 12 weeks after the end of treatment, thereby reducing both false-positive and false-negative findings, while the latter is related to the presence of undetectable microscopic residual disease [8]. Awareness and recognition of the imaging appearances of post-treatment changes is critical for the radiologist to decide whether these require conservative management or more active treatment [9].

The currently emerging method of PET/magnetic resonance imaging (MRI) is potentially an attractive alternative to PET/CT. MRI provides superior soft tissue contrast to CT and is combined with the known metabolic imaging specificity of PET [10]. This combination should prove superior in better defining tumour extent, including perineural spread and surrounding tissue infiltration [2]. Furthermore, functional MR sequences could be added to the PET/MRI protocol, e.g. diffusion-weighted imaging (DWI), as well as other multiparametric sequences, which enhance the prediction of therapy response in $\mathrm{HNC}$ [11].

The aims of our study were to: (1) evaluate the diagnostic accuracy of cePET/MRI in diagnosing recurrent $\mathrm{HNC}$ as compared to ceCT, ceMRI and cePET/CT; (2) assess the image quality concerning the prevalence of artefacts in routine clinical protocols; (3) assess whether gadolinium-enhanced MRI sequences add significant diagnostic information to PET/MRI; and (4) identify which method (PET/CT or PET/MRI) is superior to evaluate unclear FDG findings in PET.

\section{Materials and methods}

Patient population

A total of 87 adult patients ( 68 men, 19 women; mean age 63 years, range $24-90$ years) were enrolled in this prospective study. From February 2012 to March 2013, all patients referred for a clinical PET/CT examination for restaging/followup of various HNCs underwent an additional scientific MRI within a trimodality set-up. No further selection was applied to patient inclusion. Exclusion criteria were unwillingness to participate in the study, claustrophobia, MRI incompatible medical devices (e.g. cardiac pacemakers, neurostimulators, cochlear implants and insulin pumps) or possible metallic fragments in the body. This study was approved by the Institutional Ethics Committee and signed informed consent was obtained from all patients prior to the examination. Part of these patients have already been evaluated in another study [12], which compared cePET/MRI to cePET/CT for lesion detection in $\mathrm{HNC}$ with standard sequences.

\section{$\mathrm{PET} / \mathrm{CT}$ and MR imaging}

Sequential PET/CT, ceCT and ceMRI were performed on a trimodality PET/CT-MRI set-up [full ring, time-of-flight (TOF) Discovery PET/CT 690, 3 T Discovery MR750w, both GE Healthcare, Waukesha, WI, USA]. The dedicated MRand CT-compatible shuttle transfer mechanism connecting the MR and PET/CT systems allowed for PET/CT scanning free of radiofrequency (RF) coil-induced artefacts and ascertained the placement of dedicated RF coils for MRI without repositioning of the patient $[13,14]$.

Patients fasted for at least $4 \mathrm{~h}$ prior to injection of a standard dose of $4.5 \mathrm{MBq} / \mathrm{kg}$ body weight [15]. After an uptake time of $30 \mathrm{~min}$ the patients were positioned on the shuttle table in the MRI suite, and the MRI acquisition covering the region between the orbital roof and the sternal notch was started. The images were acquired by the use of a dedicated RF coil (32-Channel HD Head-Neck-Spine, GE Healthcare, Waukesha, WI, USA). The MRI protocol included T1-weighted (T1w) 3-D spoiled gradient echo pulse sequence (LAVA), T2-weighted (T2w) gradient echo sequence (IDEAL), ceT1w gradient echo sequence (IDEAL) and DWI obtained in the axial plane, followed by coronal and sagittal ceT1w gradient echo sequences (LAVA flex). Fat suppression was used for the T2w and ceT1w acquisitions. All images were acquired with a slice thickness of $4 \mathrm{~mm}$ within a total MRI scan duration of 20-25 min (scanning parameters in Table 1). The intravenously (IV) injected amount of contrast media (Omniscan, GE Healthcare) was $0.2 \mathrm{ml} / \mathrm{kg}$ body weight with an injection at a rate of $1.5 \mathrm{ml} / \mathrm{s}$.

After completion of the MRI, coils were removed and the patients were transferred to the $\mathrm{PET} / \mathrm{CT}$, still being positioned 
Table 1 MRI acquisition parameters

\begin{tabular}{|c|c|c|c|c|}
\hline Parameter & T1w LAVA & T2w IDEAL & ceT1w LAVA flex & DWI EPI-STIR \\
\hline Repetition time/echo time (ms) & $8.1 / 2.1$ & $5,188 / 80$ & $6.2 / 1.7$ & $5,500 / 66.1$ \\
\hline Echo train length & NA & 23 & NA & NA \\
\hline Flip angle $\left(^{\circ}\right)$ & 15 & 90 & 15 & 90 \\
\hline Inversion time (ms) & NA & NA & NA & 250 \\
\hline Parallel imaging acceleration factor & 2 & 2 & 2 & 2 \\
\hline Receiver bandwidth (kHz) & 83.33 & 83.33 & 166.67 & 250 \\
\hline Field of view (cm) & 24 & 24 & 24 & 24 \\
\hline Matrix & $320 \times 256$ & $320 \times 256$ & $220 \times 220$ & $320 \times 256$ \\
\hline b value $(\mathrm{s} / \mathrm{mm})$ & NA & NA & NA & 800 \\
\hline NEX & NA & NA & NA & 1 \\
\hline Number of directions & NA & NA & NA & 3 \\
\hline
\end{tabular}

T1w LAVA T1-weighted spoiled gradient echo pulse sequence, T2w IDEAL 2-point Dixon-based 3-D T2-weighted gradient echo sequence, ceT1w IDEAL 2-point Dixon-based 3-D contrast-enhanced T1-weighted gradient echo sequence, ceT1w LAVA flex 2-point Dixon-based 3-D contrast-enhanced T1-weighted gradient echo sequence, $D W I$ diffusion-weighted imaging sequence, EPI-STIR echo planar imaging-short $\tau$ inversion recovery, $N E X$ number of excitations, $N A$ not applicable

on the shuttle board. This way, the positioning of the patient within the PET/CT and the MRI was exactly the same.

After shuttle transfer to the adjacent PET/CT system, unenhanced low-dose CT and PET emission data were acquired from the mid-thigh to the vertex of the skull. Directly after the acquisition of the PET data, $70 \mathrm{ml}$ IV contrast (Visipaque 320, GE Healthcare) was injected with a speed of $3 \mathrm{ml} / \mathrm{s}$. The CT scan, with scan coverage in accordance with the MRI, was started $60 \mathrm{~s}$ after the beginning of the contrast injection. Low-dose CT and regular dose CT scans were acquired in breath-hold. Scan parameters were as follows: tube voltage $120-140 \mathrm{kV}$, tube current with automated dose modulation $60-440 \mathrm{~mA} / \mathrm{slice}$, collimation $64 \times 0.625$, pitch $0.984: 1$, rotation time $0.5 \mathrm{~ms}$, coverage speed $78 \mathrm{~mm} / \mathrm{s}$, field of view (FOV) $50 \mathrm{~cm}$, and images with a transverse pixel size of 0.625 and a slice thickness of $3.75 \mathrm{~mm}$ reconstructed in the axial, coronal and sagittal planes.

PET data were acquired in 3-D TOF mode with a scan duration of $2 \mathrm{~min}$ per bed position, an overlap of bed positions of $23 \%$ and an axial FOV of $153 \mathrm{~mm}$. The emission data were corrected for attenuation by the use of the low-dose CT $(C T A C)$ and iteratively reconstructed [matrix size $256 \times 256$, Fourier rebinning (VIP mode), VUE Point FX (3-D) with 3 iterations, 18 subsets].

\section{Image processing}

The acquired PET, ceCT and ceMRI images were transmitted to a dedicated review workstation (Advantage Workstation, Version 4.5, GE Healthcare, Milwaukee, WI, USA), which enables review of the PET, ceCT and ceMRI images side by side or in fused/overlay mode (cePET/CT, cePET/MRI). Due to the calibrated trimodality system no software-based image registration was necessary. A previously conducted study validated the image registration accuracy with less than $4 \mathrm{~mm}$ lateral misalignment between CT, PET and MRI data sets, similar to the intrinsic error assessed with phantom measurements [16].

Image analysis

Analysis was performed by a board-certified nuclear medicine physician/radiologist and a radiologist with substantial experience in PET/CT. All images were evaluated for the presence of locoregional tumour recurrence, including metastasis within the head and neck area. Lesions detected and evaluated were compared with the standard of reference, which was comprised of histopathology of the detected lesions, clinical evaluation and follow-up including all other imaging modalities. Every suspected and unclear finding (positive on PET/ CT) was confirmed by clinical local inspection and/or by histology and/or by imaging follow-up. A case-by-case decision was prospectively made in routine clinical practice. All patients (except one, see the "Results" section) had histological verification of their recurrence prior to any further therapy.

First, the ceCT and MRI without gadolinium were analysed concerning malignant lesions/tumour recurrence and metastatic lymph nodes blinded to PET findings. In a second step, the cePET/CT and non-cePET/MRI were evaluated for the presence of PET-positive or morphologically malignant lesions within the head and neck area. Lastly, the PET/MRI with ceT1w images (post contrast) were analysed to evaluate the possible advantage of the additional contrast media on lesion detection in cePET/MRI. The analysis was performed with a minimum interval of 6 weeks between each reading to minimize a possible diagnostic bias. Lesions were considered PET 
positive if their maximum standardized uptake value $\left(\mathrm{SUV}_{\max }\right)$ was at least twofold higher than the surrounding background activity.

For malignant tumours, the morphological criteria included a mass-like lesion with irregular borders and contrast enhancement. The morphological criteria for malignant lymph nodes used both on CT and MRI were enlarged lymph nodes greater than $1.0 \mathrm{~cm}$ in the short axis (and $1.5 \mathrm{~cm}$ for angular lymph nodes), cystic, a necrotic centre, a round-shaped lymph node, cluster formation, irregular boundary of the lymph node capsule and extracapsular lymph node spread.

A lesion was considered positive on ceCT or ceMRI when it presented a combination of at least two of these findings in size and morphology. For cePET/CT and cePET/MRI, a positive lesion was defined based on both morphological and functional criteria. If there were discordant findings between PET and CT/MRI, the combination of the most relevant findings (morphological and functional) was taken into account (e.g. an enlarged and irregular lymph node was considered malignant even if there was no FDG uptake). Symmetric physiological findings in the lymphatic tissue (e.g. Waldeyer's ring) or arytenoid muscle uptake were not included in these evaluations.

The lesions were additionally qualitatively classified using a likelihood evaluation ranging from 1 to 4 [1=negative (meaning no suspicious lesion detected), $2=$ possible (possibly malignant lesion), $3=$ very likely (lesion very likely to be malignant) and $4=$ definitely (malignant)]. Furthermore, in cases where lesions could not be classified and therefore were found to be unclear (e.g. due to artefacts, anatomical differentiation not entirely possible), a comparison was made between cePET/CT and cePET/MRI and an evaluation undertaken to determine whether the other imaging modality could solve the unclear finding (e.g. unclear FDG uptake on cePET/ $\mathrm{CT}$ could be solved in cePET/MR) and the reader was able to classify the lesion.

The image quality was assessed by a 3-point scale: (1) absence of relevant artefacts, (2) mild artefacts with sufficient image quality for morphological assessment or (3) substantial artefacts with insufficient image quality for further assessment.

Another assessment was done for coexistent findings not related to tumour, tumour recurrence or metastatic lymph nodes, e.g. inflammatory lesions, scars, bony lesions etc.... This evaluation was done to elaborate which of the imaging modalities offered the best diagnostic yield for the detection of these findings.

\section{Statistical analysis}

All statistical tests were performed using SPSS Statistics Version 21 (IBM, Armonk, NY, USA). A $p$ value $<0.05$ was considered statistically significant. The Wilcoxon signed ranks test was used for the comparison of the likelihood score between ceCT, cePET/CT, ceMRI and cePET/MRI. The Mann-Whitney U test was used for the comparison of grade and location of image artefacts in cePET/CT and cePET/MRI. McNemar's test was used to evaluate differences in the accuracy of the four methods. Descriptive statistics were performed to assess the frequency of significant diagnostic information provided by gadolinium and to identify which method was superior to evaluate unclear FDG findings.

\section{Results}

Among the 87 patients, 16 patients had tumour recurrence. Of those, 11 were (histologically) proven locoregional recurrence. Four patients had (lymph node) metastasis in different head and neck locations. Of those, three were histologically proven. One had a histologically proven secondary tumour and one patient had a clivus metastasis, which was defined as metastases by imaging follow-up. Additionally, eight patients underwent biopsy without any malignant finding (see below). As a primary tumour, 76 patients $(86.4 \%$ ) had a squamous cell carcinoma (SCC). Primary T staging (at primary diagnosis) was T0 (2.4 \%), T1 (28.9 \%), T2 (34.9\%), T3 (12.0\%) and T4 (21.7\%). Primary N staging (at primary diagnosis) was N0 (41.0\%), N1 (12.0\%), N2 (43.4\%) and N3 (3.6\%). Overall, $60.9 \%$ of the patients were at stage IV at primary staging. Patient data are summarized in Table 2.

The indication for current PET/CT was regular follow-up without clinical suspicion of recurrence in 82 patients and clinical suspicion for recurrence in 5 patients. The mean follow-up time after the scans evaluated here was 6.2 months (range 3.6-9.4 months). Of the patients, 67 (77\%) were alive without disease, $13(14.9 \%)$ were alive with disease, 6 (6.9\%) died of disease and $1(1.1 \%)$ was lost to follow-up.

Overall sensitivity, specificity, positive predictive value (PPV), negative predictive value (NPV) and accuracy for ceCT, cePET/CT compared to ceMRI and cePET/MRI in follow-up of HNCs are shown in Table 3. The total number of lesions detected was 117; overall 35 lesions were proven to be malignant. ceCT, ceMRI, cePET/CT and cePET/MRI accurately detected and characterized 23, 22, 30 and 30 lesions, respectively. The only difference between cePET/MRI and cePET/CT regarding accuracy was one lesion that was accurately diagnosed as post-therapeutic change on PET/MRI, while PET/CT suggested tumour recurrence. No statistically significant differences were found between cePET/CT and cePET/MRI $(p=1.0)$. See Fig. 1.

cePET/CT and cePET/MRI showed no significant differences concerning the likelihood evaluation $(p=0.405)$, but both (cePET/CT and cePET/MRI) showed higher statistically significant results when compared to ceCT and ceMRI $(p<0.001)$, as presented in Table 4. However, significantly 
Table 2 Primary and recurrent patient and tumour characteristics

\begin{tabular}{ll}
\hline Characteristics & \\
\hline No. of patients & 87 \\
Histological type, $n$ (\%) & \\
SCC & $76(86.4)$ \\
Adenocarcinoma, AdCC, neuroendocrine carcinoma & $2(2.3)$ \\
SCC-like, MEC, spindle cell-like carcinoma, & $1(1.1)$ \\
melanoma, angiosarcoma and neuroblastoma & \\
Primary site, $n$ (\%) & \\
Oropharynx & $26(29.9)$ \\
Oral cavity & $17(19.5)$ \\
Larynx & $14(16.1)$ \\
Epipharynx & $8(9.2)$ \\
Hypopharynx & $7(8.0)$ \\
Parotid space & $4(4.6)$ \\
Paranasal sinus, skin & $3(3.4)$ \\
Maxilla, CUP & $2(2.3)$ \\
Simultaneous (floor of the mouth/hypopharynx) & $1(1.1)$ \\
Follow-up proven malignancies, $n$ & 16 \\
Locoregional recurrence & 11 \\
Metastasis & 4 \\
Secondary tumour & 1 \\
Treatment, $n$ & \\
PR (with flap) & 14 \\
$\quad$ With RT & \\
$\quad$ With CT & $45(22)$ \\
$\quad$ With ND & 38 \\
No PR & 25 \\
$\quad$ With RT & 42 \\
$\quad$ With CT & 41 \\
\hline & 40 \\
\hline
\end{tabular}

Overall, at primary staging there were 88 tumours in 87 patients; 1 patient had a synchronous secondary tumour

$S C C$ squamous cell carcinoma, $M E C$ mucoepidermoid carcinoma, $A d C C$ adenoid cystic carcinoma, $P R$ primary resection, $R T$ radiation therapy, $C T$ chemotherapy, $N D$ neck dissection

different results of likelihood evaluation were found when comparing cePET/CT vs ceCT $(p=0.006)$, cePET/CT vs

Table 3 Sensitivity, specificity, PPV, NPV and accuracy of ceCT, cePET/ $\mathrm{CT}$, ceMRI and cePET/MRI based on the number of lesions detected by each method

\begin{tabular}{lllll}
\hline & ceCT & cePET/CT & ceMRI & cePET/MRI \\
\hline Sensitivity & $63.9 \%$ & $85.7 \%$ & $62.9 \%$ & $85.7 \%$ \\
Specificity & $96.3 \%$ & $92.7 \%$ & $97.6 \%$ & $93.9 \%$ \\
PPV & $88.5 \%$ & $83.3 \%$ & $91.7 \%$ & $85.7 \%$ \\
NPV & $85.7 \%$ & $93.8 \%$ & $86.0 \%$ & $93.9 \%$ \\
Accuracy & $86.3 \%$ & $90.6 \%$ & $87.2 \%$ & $91.5 \%$ \\
\hline
\end{tabular}

ceMRI $(p=0.002)$, cePET/MRI vs $\operatorname{ceCT}(p=0.022)$ and cePET/MRI vs ceMRI $(p=0.001)$.

PET/CT and PET/MRI were able to identify 46 coexistent findings not related to the tumour. These were mainly related to inflammatory diseases (such as oesophagitis, thyroiditis, mastoidopathy and sinusitis). Additionally, there were overall 34 unclear FDG findings. Of those, cePET/MRI was able to solve eleven unclear FDG findings, mainly related to superior anatomical correlation (e.g. obscured by dental artefacts); see Fig. 2. On the other hand, cePET/CT was able to solve five unclear FDG findings; those were related to questionable bone involvement; see Fig. 3.

The 18 remaining unclear FDG findings remained indeterminate and needed further evaluation [clinical inspection, imaging follow-up (10 cases) and/or histological correlation derived from biopsy ( 8 cases)] to define their outcome. None of those lesions turned out to be tumour recurrence during follow-up.

No significant difference was found between the artefact grading and hence image quality in cePET/CT and cePET/ MRI. However, there was a statistical difference in artefact location $(p=0.002)$. cePET/CT artefacts were primarily located in the suprahyoid area, while cePET/MRI artefacts were more equally distributed among the supra- and infrahyoid neck (see Tables 5 and 6).

PET/MRI with and without contrast medium yielded the same number of lesions. Thus, the addition of contrast medium did not enhance the diagnostic accuracy. However, the addition of gadolinium was found to be useful to better define the tumour margins in six patients $(6.9 \%)$.

\section{Discussion}

To our knowledge, this is one of the first studies evaluating the use of cePET/MRI in oncological follow-up of HNC patients. It proves that cePET/MRI is at least as reliable as cePET/CT in detecting locoregional recurrence of HNCs and excluding disease in healthy patients, due to its very high NPV. Furthermore, cePET/MRI is able to clarify more unclear FDG findings seen on cePET/CT than vice versa. However, the diagnostic accuracy was not significantly superior compared to cePET/CT.

\section{General aspects}

When a new imaging modality is evaluated in routine clinical practice, an obvious advantage is usually expected in comparison to existing methods. Obvious advantages are considered a higher diagnostic accuracy, comparable diagnostic accuracy at lower costs or shorter examination time, lower radiation and significant additional information, which cannot be derived by the standard method, or maybe just higher patient comfort. 

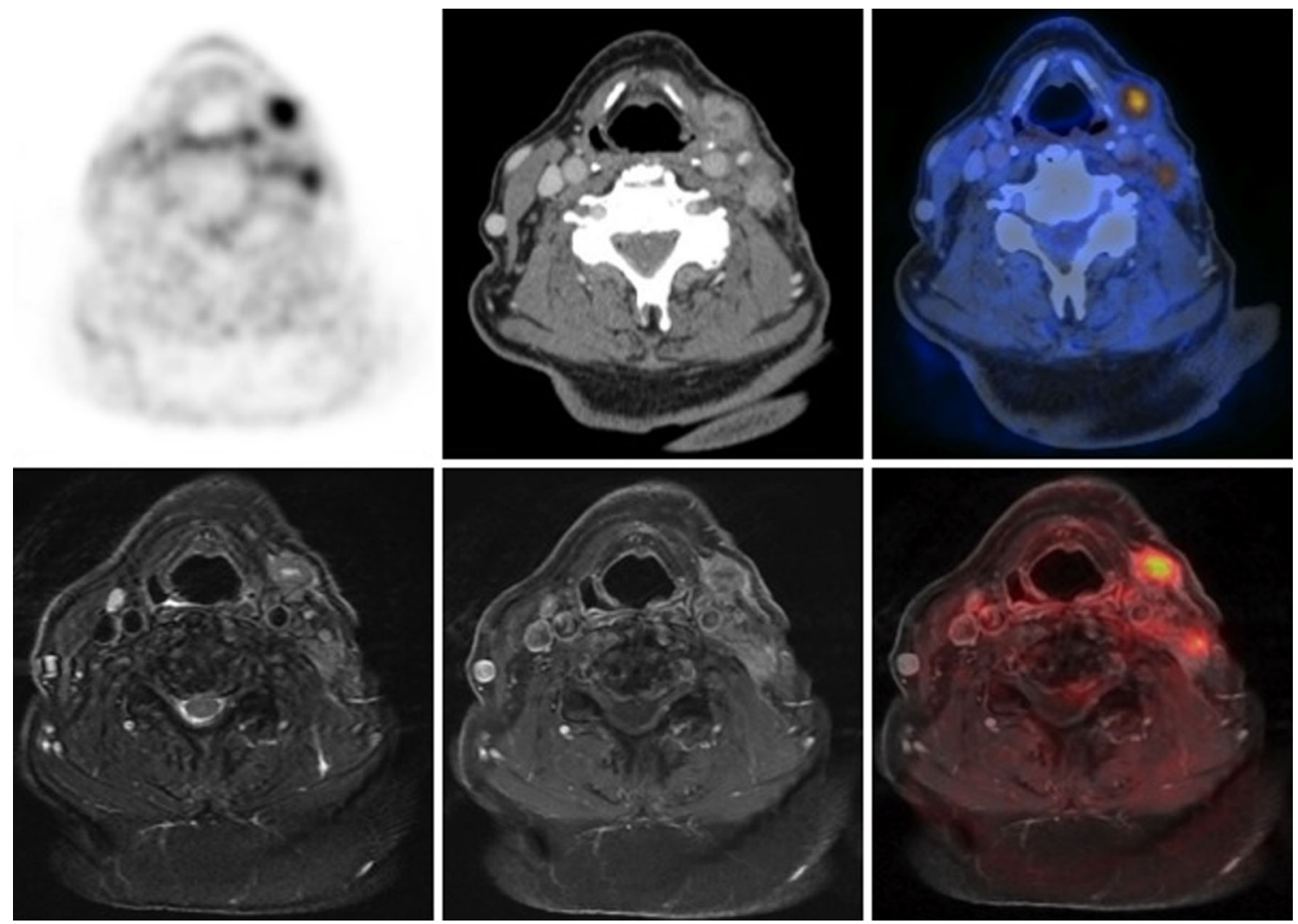

Fig. 1 An 86-year-old man with low-grade mucoepidermoid carcinoma of the left parotid gland treated with radical parotidectomy and left-sided neck dissection in cervical levels II and III as well as adjuvant

The obvious advantage of a cePET/MRI is currently the somewhat lower radiation dose, although this might be irrelevant, especially in HNC patients who receive radiotherapy. More important advantages are the known high soft tissue contrast of MRI, its signal versatility and functional/ physiological capabilities and the reduced dental artefacts as compared to PET/CT [10, 13, 17-22]. These features help in better assessing the complex anatomical distortion and tissue changes caused by surgery and radiotherapy [7, 22]. However, current disadvantages concerning its introduction into routine clinical practice are longer examination times and thus partly decreased patient comfort and procedural costs, which are certainly higher than cePET/CT or ceMRI alone. The presently non-existent reimbursement of cePET/MRI should be added to these considerations. The diagnostic accuracy therefore remains one of the major determinants for establishment

Table 4 Likelihood evaluation of tumour recurrence in each method

\begin{tabular}{lllll}
\hline & ceCT & cePET/CT & ceMRI & cePET/MRI \\
\hline 1 (no) & $67.5 \%$ & $40.2 \%$ & $68.4 \%$ & $42.7 \%$ \\
2 (possibly) & $10.3 \%$ & $29.1 \%$ & $12.0 \%$ & $27.4 \%$ \\
3 (very likely) & $8.5 \%$ & $10.3 \%$ & $12.0 \%$ & $9.4 \%$ \\
4 (definitely) & $13.7 \%$ & $20.4 \%$ & $7.6 \%$ & $20.5 \%$ \\
\hline
\end{tabular}

radiotherapy. Upper row: PET, ceCT, cePET/CT; lower row: T2w IDE$\mathrm{AL}, \mathrm{T} 1 \mathrm{w}$ after gadolinium and cePET/MRI show lymph node metastases in cervical levels II and III that were verified by histology

of this method in comparison to standard diagnostic approaches.

\section{Diagnostic accuracy}

Our study showed similar accuracy and likelihood evaluation for cePET/CT and cePET/MRI. Recent papers demonstrate that PET/MRI has the same or a slightly better accuracy when compared to PET/CT in primary staging of pancreatic [23], endometrial [24] and paediatric cancers [25]. When assessing only follow-up patients with HNCs, PET/MRI (retrospectively fused from different examinations in different scanners not connected by a shuttle for accurate coregistration) showed higher sensitivity compared to MRI alone (92 vs $67 \%$ ), which is in accordance with the results presented here [26]. It is also well established that PET/CT is superior to PET, CT or MRI alone regarding malignancy detection in the head and neck region [2, 6, 27-29].

There are only marginal differences in diagnostic accuracy between cePET/CT and cePET/MRI, however significant ones when comparing the multimodality imaging approaches with "single" modality imaging (ceCT or ceMRI). This actually shows that the metabolic PET component is the leading tool for the detection of tumour recurrence, regardless of the anatomical imaging component with which it is combined. 

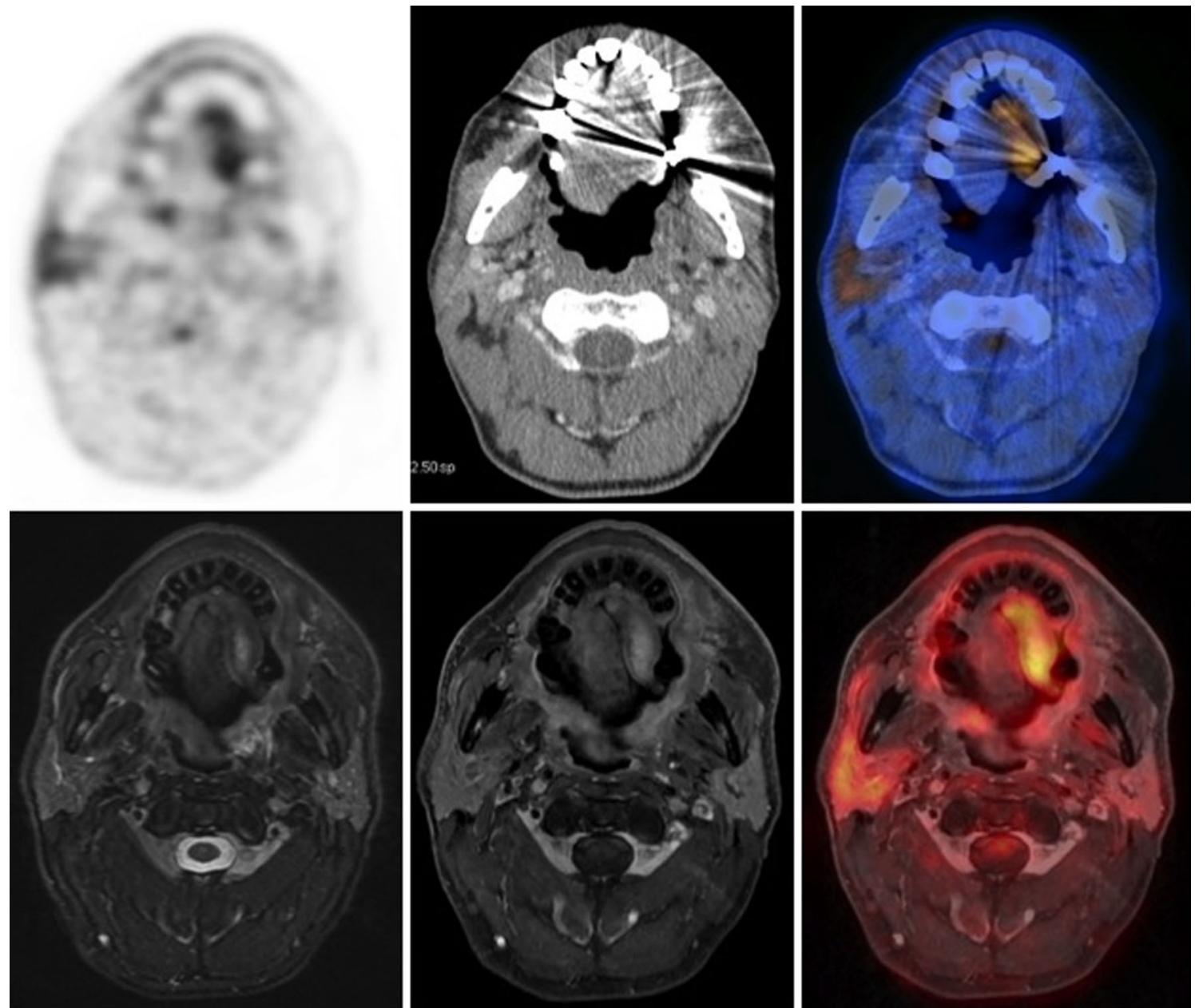

Fig. 2 A 56-year-old man with SCC of the base of the tongue on the left side (T2 N0), treated with transoral resection and reconstruction with radial flap, neck dissection in levels I-III and radiochemotherapy. Top: PET, ceCT and cePET/CT show focal FDG uptake in the left body of the tongue, suspicious for tumour recurrence. Note the dental artefacts that impair proper anatomical evaluation. Bottom: T2w IDEAL, T1w with gadolinium and cePET/MRI show focal FDG uptake in the surgical flap.

However, the study presented here concentrated on the evaluation of head and neck lesions. Other distant metastases, e.g. possible lung metastases, were not evaluated and need more focused research, especially since lung lesions are challenging to evaluate in MRI.

In regard to the specificity of multimodality imaging, although not significant, it is slightly lower, reflecting the posttherapeutic setting where different focal and/or diffuse metabolic findings can account for false-positive findings (see also section below).

\section{Unclear FDG findings}

Since the PET component is the same for cePET/CT and cePET/MRI, both methods detected the same number of unclear FDG findings. However, based on its known higher soft tissue contrast, cePET/MRI was better able to accurately
T2w IDEAL shows high intensity on the left side of the postoperative tongue, indicating increased water or fat caused by the tongue denervation after neck dissection. Contrast enhancement is seen in this area probably because of prominent interstitial space between atrophic tongue muscle tissues. FDG uptake probably indicates the presence of inflammation that might be due to radiation therapy. Absence of tumour verified by histology

clarify these findings and depict the underlying pathology. Most cases were related to focal FDG uptake without anatomical correlation due to dental artefacts on ceCT or lesions with low soft tissue contrast (e.g. tongue uptake). Additionally, one case of perineural tumour spread could only be identified on cePET/MRI. cePET/MRI might therefore have certain advantages in the evaluation of anatomically complex postoperative cases; however, concerning the overall accuracy we could not demonstrate such a result in our patient populations. As this assumption is in line with the current literature, it is merely reflected in our likelihood evaluation. In a population with treated nasopharyngeal carcinoma, Glastonbury and Salzman have shown that MRI is recommended in patients with new trismus to look for evidence of perineural tumour or a recurrent mass and distinguish this from radiation-induced muscle inflammation [17]. Other papers reinforce the need for MRI in detecting perineural spread $[2,7]$. On the other hand, 

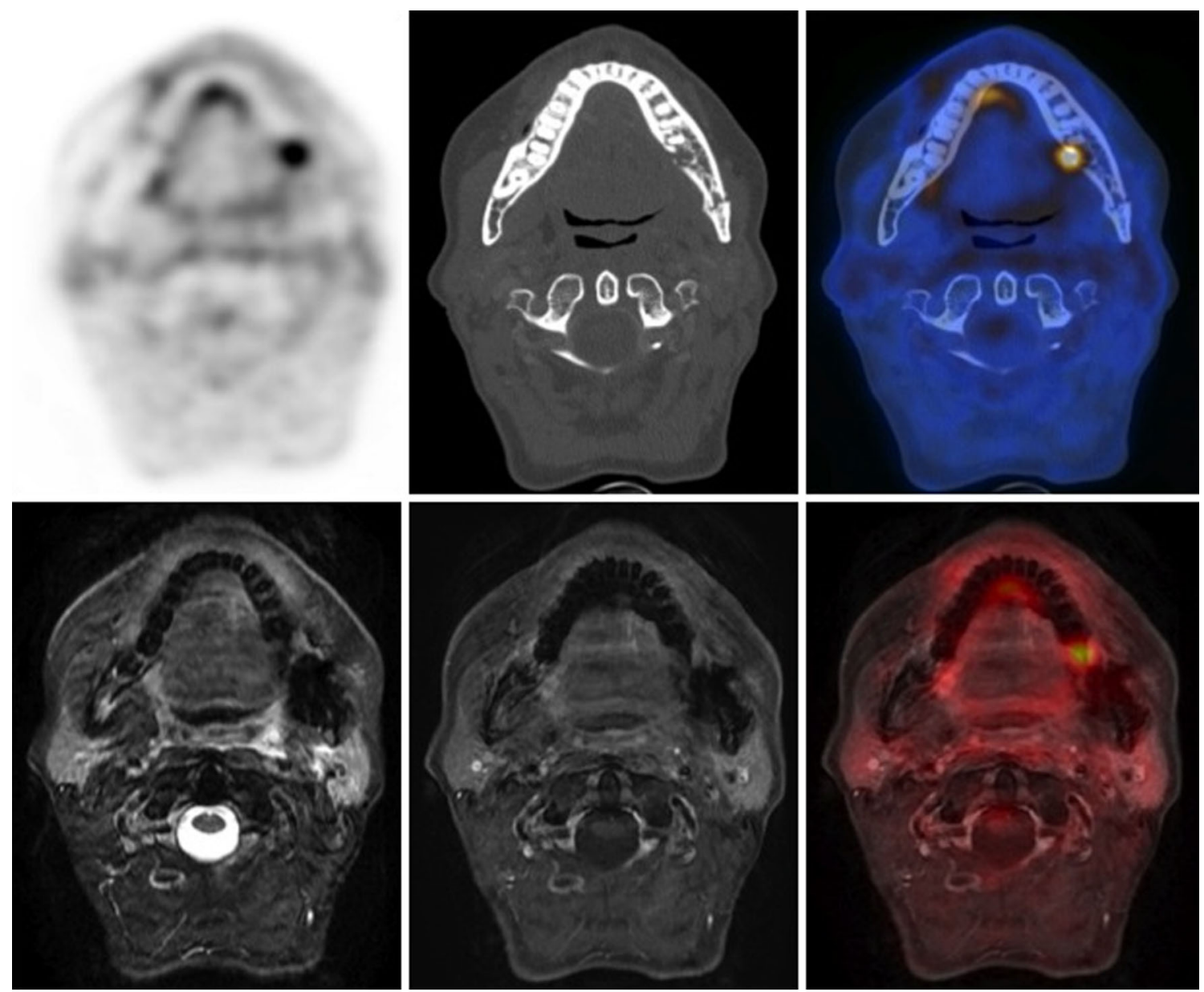

Fig. 3 A 48-year-old woman with SCC of the left tonsil cT2 cN1 cM0, treated with radiochemotherapy with a dose of 70 Gy. Top: PET, ceCT (bone window), cePET/CT showing FDG uptake in a lytic lesion in the mandible on the left side. Bottom: T2w IDEAL, T1w with gadolinium

cePET/CT was better suited to identify osseous lesions, remaining as an excellent diagnostic imaging method to detect bone involvement, like radio-osteonecrosis and metastatic disease. Other studies also have shown that PET/CT is partly superior to PET/MRI for the detection and evaluation of conspicuous bone lesions [20,30]. Our own study showed four osseous lesions better detected by cePET/CT.

Image quality

Regarding image quality, both cePET/MRI and cePET/CT received similar scoring, however with regional differences. The oropharyngeal area was more often obscured in cePET/

Table 5 Artefacts' grade by method

\begin{tabular}{lll}
\hline Grade & cePET/CT & cePET/MRI \\
\hline No & $70.1 \%$ & $72.4 \%$ \\
Mild & $21.8 \%$ & $24.2 \%$ \\
Substantial & $8.1 \%$ & $3.4 \%$ \\
\hline
\end{tabular}

and cePET/MRI showing the FDG uptake in the mandible on the left side, but motion and dental artefacts in the oropharynx impair proper analysis by MR images. Biopsy confirmed the lesion to be radiogenic ulcer

CT due to dental artefacts, suggesting that for restaging of cancer of the oropharynx and the oral cavity cePET/MRI might be the best option. Previous studies also suggested that PET/MRI could be useful when evaluating the oropharynx (which contains up to $25 \%$ of HNC), since there are several MRI sequences available that minimize dental artefacts [13, 19, 31]. Even in the "conventional" MR sequences used in our study, dental artefacts were significantly reduced. In the infrahyoid neck, however, cePET/MRI showed inferior image quality compared to cePET/CT, mainly owing to the patient's movement, swallowing and carotid pulsation. Thus, patients

Table 6 Artefacts by anatomical site

\begin{tabular}{lll}
\hline Anatomical site, $\%$ & cePET/CT & cePET/MRI \\
\hline Nasopharynx & 0 & $4.0 \%$ \\
Oropharynx & $96.2 \%$ & $56.0 \%$ \\
Hypopharynx/larynx & $3.8 \%$ & $40.0 \%$ \\
\hline
\end{tabular}

${ }^{\mathrm{a}}$ One patient had artefacts in two sites 
with suspected recurrent cancer in the hypopharynx or larynx might be better evaluated with cePET/CT. There are several techniques to reduce these artefacts in MRI that, however, require prolonged scanning times, which might not be applicable in all patients (patient comfort), or feasible in a tight clinical schedule, especially if cePET/MRI is compared with cePET/CT as a whole-body imaging method.

Barral and co-workers have shown that MRI requires long examination times and therefore is vulnerable to involuntary and physiological motion, since patients often swallow and cough during the scan, and the carotid arteries are typically within the FOV, causing pulsatile flow artefacts [26, 32]. Motion-related artefacts in the nasopharynx are rare in both methods because of its fixed position in the skull base and the pharyngobasilar fascia. In this area, both methods are equally useful, as also shown in the literature [12].

The use of gadolinium did not add any relevant information concerning lesion detection or diagnostic accuracy. Thus, no contrast needs to be injected for lesion detection, particularly in patients with contraindications to contrast administration. However, gadolinium has been found to be useful in delineating the tumour margins. This might be important for radiotherapy planning, particularly in a recurrence setting where patients frequently get radiotherapy or for planning surgical intervention, since the relation of the tumour to adjacent structures is much better delineated. Prestwich and coworkers showed that the use of MRI is beneficial for delineating adjacent organs at risk that are poorly visualized on CT, for example the optic nerves and chiasm, brainstem, spinal cord, brachial plexus and parotid glands [33].

\section{Limitations}

The short follow-up time following imaging evaluation is certainly a limitation of our study; however, PET/MRI has only recently become available and in this regard our results should be viewed as preliminary. Longer follow-up times might be beneficial, e.g. to clarify unclear FDG findings. Moreover, despite the large number of patients included in the study, only a small number proved to have tumour recurrence. However, this on the other hand contributes to the evaluation of the NPV.

\section{Conclusion}

cePET/MRI is slightly superior compared to cePET/CT to evaluate areas of unclear FDG findings in follow-up of patients after HNC. cePET/MRI might also be the preferred imaging approach for the evaluation of the oropharynx and the oral cavity based on a lower incidence of artefacts. In turn, bone involvement is possibly better seen with cePET/CT. Overall, there were no statistically significant differences between the two methods concerning their diagnostic accuracy in follow-up of patients after HNC.

Disclosures This research project was supported by an institutional research grant from GE Healthcare. Patrick Veit-Haibach received IIS Grants from Bayer Healthcare and Siemens Medical Solutions and speaker fees from GE Healthcare. Gustav von Schulthess is a grant recipient from GE Healthcare and receives speaker fees from GE Healthcare.

Conflicts of interest The other authors declare no other conflicts of interest.

\section{References}

1. Rangaswamy B, Fardanesh MR, Genden EM, Park EE, Fatterpekar $\mathrm{G}$, Patel $\mathrm{Z}$, et al. Improvement in the detection of locoregional recurrence in head and neck malignancies: F-18 fluorodeoxyglucose-positron emission tomography/computed tomography compared to high-resolution contrast-enhanced computed tomography and endoscopic examination. Laryngoscope 2013;123: 2664-9.

2. Sadick M, Schoenberg SO, Hoermann K, Sadick H. Current oncologic concepts and emerging techniques for imaging of head and neck squamous cell cancer. GMS Curr Top Otorhinolaryngol Head Neck Surg 2012;11:Doc08.

3. Herrmann K, Krause BJ, Bundschuh RA, Dechow T, Schwaiger M. Monitoring response to therapeutic interventions in patients with cancer. Semin Nucl Med 2009;39(3):210-32.

4. Abgral R, Querellou S, Potard G, Le Roux PY, Le Duc-Pennec A, Marianovski R, et al. Does 18F-FDG PET/CT improve the detection of posttreatment recurrence of head and neck squamous cell carcinoma in patients negative for disease on clinical follow-up? J Nucl Med 2009;50(1):24-9.

5. Ul-Hassan F, Simo R, Guerrero-Urbano T, Oakley R, Jeannon JP, Cook GJ. Can (18)F-FDG PET/CT reliably assess response to primary treatment of head and neck cancer? Clin Nucl Med 2013;38(4): 263-5.

6. Schöder H, Fury M, Lee N, Kraus D. PET monitoring of therapy response in head and neck squamous cell carcinoma. J Nucl Med 2009;50 Suppl 1:74S-88S.

7. Saito N, Nadgir RN, Nakahira M, Takahashi M, Uchino A, Kimura F, et al. Posttreatment CT and MR imaging in head and neck cancer: what the radiologist needs to know. Radiographics 2012;32:1261-82.

8. Castaldi P, Leccisotti L, Bussu F, Miccichè F, Rufini V. Role of (18)FFDG PET-CT in head and neck squamous cell carcinoma. Acta Otorhinolaryngol Ital 2013;33(1):1-8.

9. Offiah C, Hall E. Post-treatment imaging appearances in head and neck cancer patients. Clin Radiol 2011;66(1):13-24.

10. Platzek I, Beuthien-Baumann B, Schneider M, Gudziol V, Langner J, Schramm G, et al. PET/MRI in head and neck cancer: initial experience. Eur J Nucl Med Mol Imaging 2013;40(1):6-11.

11. Chawla S, Kim S, Dougherty L, Wang S, Loevner LA, Quon H, et al. Pretreatment diffusion-weighted and dynamic contrast-enhanced MRI for prediction of local treatment response in squamous cell carcinomas of the head and neck. AJR Am J Roentgenol 2013;200(1):35-43.

12. Kuhn F, Huellner M, von Schulthess G, Veit-Haibach P. Comparison of contrast enhanced PET/MRI and contrast enhanced PET/CT in patients with head and neck cancer. J Nucl Med 2013;54(Suppl 2): 515. 
13. Veit-Haibach P, Kuhn FP, Wiesinger F, Delso G, von Schulthess G. PET-MR imaging using a tri-modality PET/CT-MR system with a dedicated shuttle in clinical routine. MAGMA 2013;26:25-35.

14. Kuhn FP, Crook DW, Mader CE, Appenzeller P, von Schulthess GK, Schmid DT. Discrimination and anatomical mapping of PET-positive lesions: comparison of CT attenuation-corrected PET images with coregistered MR and CT images in the abdomen. Eur J Nucl Med Mol Imaging 2013;40(1):44-51.

15. Boellaard R, O'Doherty MJ, Weber WA, Mottaghy FM, Lonsdale $\mathrm{MN}$, Stroobants SG, et al. FDG PET and PET/CT: EANM procedure guidelines for tumour PET imaging: version 1.0. Eur J Nucl Med Mol Imaging 2010;37(1):181-200.

16. Kuhn F, Wiesinger F, Wollenweber S, Samarin A, Von Schulthess G, Schmid D. Sequential integrated PET/CT-MR system: comparison of image registration accuracy of PET/CT versus PET/MR. Melbourne, Australia: International Society for Magnetic Resonance in Medicine (ISMRM); 2012

17. Glastonbury CM, Salzman KL. Pitfalls in the staging of cancer of nasopharyngeal carcinoma. Neuroimaging Clin N Am 2013;23(1):925.

18. Buchbender C, Heusner TA, Lauenstein TC, Bockisch A, Antoch G. Oncologic PET/MRI, part 1: tumors of the brain, head and neck, chest, abdomen, and pelvis. J Nucl Med 2012;53(6):928-38.

19. von Schulthess GK, Kuhn FP, Kaufmann P, Veit-Haibach P. Clinical positron emission tomography/magnetic resonance imaging applications. Semin Nucl Med 2013;43(1):3-10.

20. Appenzeller P, Mader C, Huellner MW, Schmidt D, Schmid D, Boss $\mathrm{A}$, et al. PET/CT versus body coil PET/MRI: how low can you go? Insights Imaging 2013;4(4):481-90.

21. Castelijns JA. PET-MRI in the head and neck area: challenges and new directions. Eur Radiol 2011;21(11):2425-6.

22. Bhargava $\mathrm{P}$, Rahman S, Wendt J. Atlas of confounding factors in head and neck PET/CT imaging. Clin Nucl Med 2011;36(5):e20-9.

23. Nagamachi S, Nishii R, Wakamatsu H, Mizutani Y, Kiyohara S, Fujita $\mathrm{S}$, et al. The usefulness of (18)F-FDG PET/MRI fusion image in diagnosing pancreatic tumor: comparison with (18)F-FDG PET/ CT. Ann Nucl Med 2013;27(6):554-63.

24. Kitajima K, Suenaga Y, Ueno Y, Kanda T, Maeda T, Takahashi S, et al. Value of fusion of PET and MRI for staging of endometrial cancer: comparison with (18)F-FDG contrast-enhanced PET/CT and dynamic contrast-enhanced pelvic MRI. Eur J Radiol 2013;82(10): $1672-6$.

25. Pfluger T, Melzer HI, Mueller WP, Coppenrath E, Bartenstein P, Albert MH, et al. Diagnostic value of combined (18)F-FDG PET/ MRI for staging and restaging in paediatric oncology. Eur J Nucl Med Mol Imaging 2012;39(11):1745-55.

26. Nakamoto $Y$, Tamai K, Saga T, Higashi T, Hara T, Suga T, et al. Clinical value of image fusion from MR and PET in patients with head and neck cancer. Mol Imaging Biol 2009;11(1):46-53.

27. Ishikita T, Oriuchi N, Higuchi T, Miyashita G, Arisaka Y, Paudyal B, et al. Additional value of integrated PET/CT over PET alone in the initial staging and follow up of head and neck malignancy. Ann Nucl Med 2010;24(2):77-82.

28. Passero VA, Branstetter BF, Shuai Y, Heron DE, Gibson MK, Lai SY, et al. Response assessment by combined PET-CT scan versus CT scan alone using RECIST in patients with locally advanced head and neck cancer treated with chemoradiotherapy. Ann Oncol 2010;21(11):2278-83.

29. Kim SY, Kim JS, Yi JS, Lee JH, Choi SH, Nam SY, et al. Evaluation of $18 \mathrm{~F}-\mathrm{FDG}$ PET/CT and CT/MRI with histopathologic correlation in patients undergoing salvage surgery for head and neck squamous cell carcinoma. Ann Surg Oncol 2011;18(9):2579-84.

30. Samarin A, Burger C, Wollenweber SD, Crook DW, Burger IA, Schmid DT, et al. PET/MR imaging of bone lesions-implications for PET quantification from imperfect attenuation correction. Eur J Nucl Med Mol Imaging 2012;39(7):1154-60.

31. Nassenstein K, Veit-Haibach P, Stergar H, Gutzeit A, Freudenberg L, Kuehl H, et al. Cervical lymph node metastases of unknown origin: primary tumor detection with whole-body positron emission tomography/computed tomography. Acta Radiol 2007;48(10): 1101-8.

32. Barral JK, Santos JM, Damrose EJ, Fischbein NJ, Nishimura DG. Real-time motion correction for high-resolution larynx imaging. Magn Reson Med 2011;66(1):174-9.

33. Prestwich RJ, Sykes J, Carey B, Sen M, Dyker KE, Scarsbrook AF. Improving target definition for head and neck radiotherapy: a place for magnetic resonance imaging and 18-fluoride fluorodeoxyglucose positron emission tomography? Clin Oncol (R Coll Radiol) 2012;24(8):577-89. 